\title{
GENETICS and G3: Community-Driven Science, Community-Driven Journals
}

M OST scholarly societies publish journals for the betterment of the community, among myriad reasons. Yet in this era of rapidly proliferating publications, societies and their journals offer many benefits for constituents, including affordable meetings, travel awards, public outreach, support for educators, science advocacy, dissemination of scientific discoveries via journals, the fostering of community, boards of directors elected by their peers, and a long-term view.

At GENETICS and G3: Genes|Genomes|Genetics, we believe that society journals offer special value not found elsewhere: a voice and vehicle for practicing scientists to move science forward. The GSA journals have done this in several ways.

As peer scientists and editors, we recognize that science advances faster when the development of new methods is accompanied by clear descriptions of how to implement these methods, along with the data that benchmark the new approaches against existing ones (de Koning and McIntyre 2012).

In 2010 the GSA journals created a revised data policy that requires public accessibility of all the data used to support the conclusions described in an article (McIntyre 2010). The Editors felt a policy was necessary to ensure that analyses based on genomic data could be repeated just as easily, for example, as those based on PCR experiments; while no one today would publish the results of a PCR experiment without providing the sequences of the primers, this was not always the case.

Even as shrinking methods sections plague many journals, we have expanded requirements for methodological details. As editors, we encourage our authors to make their findings as reproducible as possible, in part because we heard the community pleading for more information. As reviewers, we appreciate having the information needed to verify and reproduce and extend results. And as authors, we sometimes struggle to conform to our own high standard. During this past year, although an increasing number of

Copyright (C) 2014 by the Genetics Society of America

doi: 10.1534/genetics.114.169151

Available freely online. scientific journals have, with much fanfare, increased their demands for open access to data, the GSA journals have in fact quietly led the way as early proponents of open data policies.

Soon after we released our revised policy, we realized that requiring raw data was not enough. Deeper thought and discussion revealed that analysis programs, detailed bioinformatics methods, and full result files were also needed to create robust publications. How though to ensure that the data would be available in perpetuity? Unfortunately, local websites, while well intentioned, often suffer from lack of maintenance over time, with broken links impeding readers' ability to access data in the long term (Vines et al. 2014). To accommodate the increasing volume of data, GENETICS and G3 encourage deposits with third-party data repositories like Dryad, FigShare, GEO, and others. We also encourage the provision of such data as supporting information.

In the spirit of serving the community, we started collections of articles on contemporary topics of interest organized across the two journals, offering a wider platform and higher visibility to each individual article (http://www.genetics.org/ site/misc/GenomicSelection.xhtml; http://g3journal.org/site/ misc/MouseGeneticResources.xhtml).

The value of such efforts is most apparent when a single topic quickly captures the interest of our community. In these cases, having common data sets to work with becomes critical. In February 2012, the GSA journals featured a focus on the mouse Collaborative Cross, which underscored the role of society journals in promoting such community endeavors (McIntyre and de Koning 2012). GENETICS and G3 have gone on to encourage discussion in genomic selection (de Koning and McIntyre 2012), sex determination (http://www. genetics.org/site/misc/GeneticsOfsex.xhtml), and immunity (http://www.genetics.org/site/misc/GeneticsOfImmunity.xhtml), and we continue to promote and present literature on these topics. Please let us know if there are challenges and central questions in your community that need a venue for marshalling discussion. 


\section{GENETICS and G3 Today: Multiparental Populations Collection}

In the continued spirit of hosting such scholarly resources, the current issues of GENETICS and G3 highlight articles on the study of multiparental populations (\#MPP). As the limitations of two-parent cross designs have become obvious, we are struggling with analysis of experimental populations that have more than two segregating alleles. The articles in the MPP collection, along with their data and transparent methods, should stimulate discussion and, we hope, move the field forward.

The realization of this first group of MPP articles exemplifies the ability of community-driven journals to support communitydriven research. The idea for this initiative originated during a workshop on Multiparent Advanced Generation InterCross (MAGIC) populations (http://mus.well.ox.ac.uk/ 19genomes/MAGIC-WORKSHOP/) in 2013 that was coorganized by one of our Associate Editors, B. Emma Huang. With the help of Associate Editors Fred van Eeuwijk and Alain Charcosset, the journals issued a call for articles (http:// www.genetics.org/site/misc/CallForPapersMultiParental.xhtml). The submissions were managed by additional editors (the authors of this editorial, as well as Jim Birchler, Steve Chenoweth, Rebecca W. Doerge, Jim Holland, B. Emma
Huang, Brian Yandell, and senior editor Krista Nichols) and nearly 70 referees.

Such an effort-indeed, the very existence of society journals themselves-would be hard to envisage without the support and leadership of an engaged community of practicing scientists, who themselves understand what is necessary to "do science" and to thrive in today's current climate. When we commit to publishing reproducible science, the synergy creates a body of long-standing work that is greater than the sum of its parts. We invite you to submit your own manuscripts for consideration in the rolling MPP collection as well as others, have your own voice heard, and advance discovery and discussion in your community.

\section{Literature Cited}

de Koning, D.-J., and L. McIntyre, 2012 Setting the standard: a special focus on genomic selection in GENETICS and G3. G3 2: 423.

McIntyre, L., 2010 Data: The Foundation of Science. Genetics 184: 1 .

McIntyre, L., and D.-J. de Koning, 2012 Facilitating discovery: the role of society journals in collaborative science. Genetics 190: 285-286.

Vines, T. H., A. Y. K. Albert, R. L. Andrew, F. Débarre, D. G. Bock et al., 2014 The availability of research data declines rapidly with article age. Curr. Biol. 24: 94-97.

Dirk-Jan de Koning, Deputy Editor, G3: Genes | Genomes | Genetics Swedish University of Agricultural Sciences Lauren M. McIntyre, Senior Editor, GENETICS

University of Florida 